Maikel Chao Parapar*

\title{
En cualquier caso. Marcadores de distanciamiento en textos científicos en español
}

\section{En cualquier caso. Discourse markers and distance in scientific texts in Spanish}

https://doi.org/10.1515/soprag-2020-0017

Published online May 31, 2021

Resumen: El objetivo del presente trabajo es observar el empleo de los marcadores en cualquier caso, en todo caso, de todos modos y de todas maneras en el discurso científico. Se ha estudiado su uso en un corpus de textos de cuatro ciencias, representantes de las cuatro áreas en las que se divide el conocimiento: Ciencias, Ciencias Aplicadas, Ciencias Sociales y Humanidades. Se presta especial atención a la función que puedan cumplir dentro de lo que se ha dado a conocer como metadiscurso y al uso que el autor pueda hacer de estos mecanismos para la comunicación con su futuro lector. Los resultados muestran que el uso de estos marcadores discursivos ayuda a los autores de los textos científicos, en mayor o menor medida, a persuadir a los lectores. Por ello, los datos obtenidos justifican la inclusión de los marcadores del discurso dentro de un modelo metadiscursivo para el español científico.

Palabras clave: discurso científico, marcadores discursivos, metadiscurso

Abstract: The aim of this study is to understand the use of the markers en cualquier caso, en todo caso, de todos modos and de todas maneras in scientific discourse. These discourse markers are observed in a corpus that is composed of texts from four scientific fields that represent the four areas in which knowledge is divided: Sciences, Applied Sciences, Social Sciences, and Humanities. Special attention is given to the function these markers fulfill within what is known as metadiscourse and to the use the author may make of these mechanisms to

*Corresponding author: Maikel Chao Parapar, Facultad de Filología Calle Lisboa 7, Universidade da Coruña, A Coruna, 15008, Spain, E-mail: maikel.dcp@gmail.com. https://orcid.org/00000002-7458-1739

Ә Open Access. @ 2021 Maikel Chao Parapar, published by De Gruyter. (๕) BY-NC-ND This work is licensed under the Creative Commons Attribution-NonCommercial-NoDerivatives 4.0 International License. 
communicate with his/her future reader. Results show that these discourse markers contribute to persuade readers to a greater or lesser extent. Therefore, the obtained data justify the inclusion of discourse markers in a metadiscoursive model in order to study scientific Spanish.

Keywords: discourse markers, metadiscourse, scientific discourse

\section{Introducción}

Suele señalarse la década de los 60 como el inicio de los estudios a nivel supraoracional. Desde entonces, elementos como los marcadores del discurso han gozado de una gran popularidad en el campo de la lingüística (Fuentes Rodríguez, 2009; Garcés Gómez, 2008, 2013; Loureda y Acín Villa, 2010; Martín Zorraquino y Montolío Durán, 1998; Martín Zorraquino y Portolés, 1999; Montolío Durán, 2001). Aunque la mayoría de los trabajos que se han realizado hasta el momento se centran más en el lenguaje coloquial (Acín Villa, 2001, 2019; Briz, 1993a, 1993b, 1994, 1998; Boyero Rodríguez, 2002; Casado Velarde, 1991; Cortés Rodríguez, 1991; Fuentes Rodríguez, 1990), también pueden encontrarse en menor medida estudios de marcadores del discurso en lenguajes especializados (Eisner y MontemayorBorsinger, 2014; Wiefling y Montemayor-Borsinger, 2014). Utilizaremos para nuestro trabajo el término de marcadores del discurso, así como la definición propuesta por Martín Zorraquino y Portolés (1999). Por lo que evitaremos emplear terminología de obras como la Nueva Gramática de la Lengua Española (RAE, 2009), que habla de conectores discursivos, puesto que el término conector, cuyo origen reside en la lógica, se refiere solo a operadores que enlazan dos proposiciones (Escandell Vidal, 2004), mientras que el que nosotros utilizamos recoge un abanico más amplio de relaciones en el discurso (Garcés Gómez, 2008; Portolés, 2014).

Aclarado este punto, debemos indicar que, aunque nuestro objetivo es estudiar los marcadores en cualquier caso, en todo caso, de todos modos y de todas maneras en el discurso de especialidad, no vamos a profundizar en todo lo que se refiere a nociones básicas de esta clase de unidades lingüísticas más allá de las propias piezas que estudiamos aquí. Dada la gran cantidad de estudios que se pueden encontrar al respecto, consideramos más oportuno centrar nuestros esfuerzos en el marco teórico en el que se enmarcan nuestros trabajos, esto es, el concepto, características y clasificaciones del metadiscurso. De este modo, pretendemos observar cómo se comportan en el discurso académico-científico, lo que nos permitirá, además, concluir si es pertinente o no introducir los marcadores del discurso como una de las categorías de nuestro modelo para el análisis del 
metadiscurso (§2.2). Partimos de la hipótesis de que estos marcadores serán relevantes en el discurso de las Humanidades, pero debemos estudiar si lo son en disciplinas de las otras ramas del conocimiento.

Por su parte, en nuestro trabajo hablaremos de académico-científico cuando queramos englobar estas dos áreas que, en principio, entendemos como dos grupos diferentes de un mismo lenguaje de especialidad. Esta separación, que ya podemos encontrar en estudios como los desarrollados por Parodi (2008a, 2008b, 2008c, 2010, 2015), se debe, en nuestro caso, al gran número de trabajos que han permitido descubrir un abanico muy amplio de géneros discursivos como las notas de evaluación por parte del profesorado, informes de laboratorio, pósteres, mesas redondas, los trabajos de final de carrera o máster, la tesis doctoral o el artículo de investigación. Todos estos pueden agruparse bajo la etiqueta académico-científico, sin embargo, entre los primeros y los últimos hay una gran diferencia y en lo que a uso de la lengua se refiere. Por este motivo, diferenciamos entre discursos académicos y discursos científicos, dependiendo de las características lingüístico-contextuales en las que se produzca el discurso. De este modo, esa gran cantidad de subgéneros se reparten en dos que comparten ciertos rasgos, pero no son totalmente iguales. Para hablar de características simultáneas utilizaremos el término englobador que indicábamos al comienzo del párrafo, es decir, discurso académico-científico.

Tras esta breve introducción a nuestro trabajo, el segundo apartado servirá para exponer el estado de la cuestión en lo que a metadiscurso se refiere, es decir, definición, características y modelos. En el tercero expondremos la metodología empleada para llevar a cabo el estudio. Para terminar, en los dos últimos apartados se recogen los resultados obtenidos y las conclusiones a las que se ha llegado, respectivamente.

\section{Metadiscurso}

\subsection{Antecedentes}

En las últimas décadas, el ámbito anglosajón ha aportado un gran número de publicaciones cuyo objetivo era, y sigue siendo, conocer los mecanismos lingüísticos que permiten que los interlocutores de los diferentes discursos académicos y científicos se comuniquen, así como conocer qué papeles adoptan los autores y los lectores de estos textos. Uno de los elementos clave en estos estudios es el metadiscurso, término acuñado por Zellig S. Harris (1959) en una publicación dedicada a la estructura de la información en textos científicos desde la gramática generativa transformacional. Presenta el concepto de metadiscourse kernels como expresiones que “dicen algo sobre el contenido principal” (Harris, 1970 [1959]: 
944). Puede que la escasa descripción y caracterización del concepto sea el motivo por el cual haya que esperar dos décadas para encontrar un trabajo en el que se aporte una primera definición del metadiscurso: "escritura sobre la escritura" (Williams, 1990 [1981]).

Sucesivos estudios intentarán aportar una definición más exacta del concepto (Crismore, Markkanen, Steffensen, 1993; Vande Koople, 1985), pero habrá que esperar hasta finales de los años noventa para que el lingüista británico Ken Hyland (1998, 2000, 2005, 2010; Hyland y Tse 2004) desarrolle una compleja teoría en la que propone no solo una renovada definición, sino también una categorización de los recursos que este concepto abarca. Para Hyland, el metadiscurso es "una categoría funcional que se manifiesta a través de una serie de procedimientos lingüísticos por medio de los cuales el autor del texto organiza su discurso, se dirige al lector o expresa su opinión sobre lo escrito" (Hyland, 2000: 109). En un trabajo posterior Hyland y Tse (2004) establecen tres principios claves para determinar si un elemento cumple una función metadiscursiva:

I. En primer lugar, retoman la característica de los primeros estudios sobre el metadiscurso de Harris (1959), J. M. Williams (1990 [1981]) y Vande Koople (1985): las expresiones metadiscursivas no forman parte del contenido proposicional. No obstante, advierten, debemos tomar este primer principio con cuidado, pues en muchas ocasiones el margen entre lo proposicional y lo metadiscursivo no se puede apreciar con claridad.

II. La relación autor-lector-texto: el metadiscurso se considera un elemento esencial para una comunicación satisfactoria. Por ello, es importante para el autor del artículo científico hacer partícipe al futuro público lector. Es así como las expresiones metadiscursivas deben convertirse en un enlace de unión entre los participantes del discurso y, en ocasiones, entre estos y el propio texto.

III. La distinción de lo interno y lo externo: una de las principales claves para la localización de una expresión metadiscursiva es su capacidad para establecer no solo conexiones entre partes de un mismo texto (metadiscurso textual), sino también entre el discurso y el propio acto de escritura, por ejemplo.

En la Tabla 1 encontramos la clasificación que presentan Hyland y Tse (2004) de las expresiones metadiscursivos. Como podemos apreciar, se presentan dos tipos de recursos: interactive (denominado también textual), elementos que se centran en guiar al lector a través del discurso, e interactional (también llamado interpersonal), recursos enfocados a hacer partícipe al futuro público.

Posteriormente, Hyland publicará una serie de trabajos en los que ampliará el modelo que hemos visto en la tabla anterior (Hyland y Tse, 2004). La primera diferencia que encontramos es la división bipartita del grupo que anteriormente 
Tabla 1: Modelo de metadiscurso de Hyland y Tse (Fuente: Hyland y Tse, 2004:169).

\begin{tabular}{|c|c|c|}
\hline Category & Function & Examples \\
\hline $\begin{array}{l}\text { Interactive } \\
\text { resources }\end{array}$ & Help to guide reader through the text & \\
\hline Transition & $\begin{array}{l}\text { Express semantic relation between } \\
\text { main clauses }\end{array}$ & In addition/but/thus/and \\
\hline Frame markers & $\begin{array}{l}\text { Refer to discourse acts, sequences, or } \\
\text { text stages }\end{array}$ & $\begin{array}{l}\text { Finally/to conclude/my purpose } \\
\text { here is to }\end{array}$ \\
\hline Endophoric markers & $\begin{array}{l}\text { Refer to information in other parts of the } \\
\text { text }\end{array}$ & Noted above/see Fig./in Section 2 \\
\hline Evidentials & $\begin{array}{l}\text { Refer to source of information from } \\
\text { other texts }\end{array}$ & According to $X /(Y, 1990) / Z$ states \\
\hline Code glosses & $\begin{array}{l}\text { Help readers grasp functions of idea- } \\
\text { tional material }\end{array}$ & $\begin{array}{l}\text { Namely/e.g./such as/in other } \\
\text { words }\end{array}$ \\
\hline $\begin{array}{l}\text { Interactional } \\
\text { resources }\end{array}$ & Involve the reader in the argument & \\
\hline Hedges & $\begin{array}{l}\text { Withhold writer's full commitment to } \\
\text { proposition }\end{array}$ & Might/perhaps/possible/about \\
\hline Boosters & $\begin{array}{l}\text { Emphasize force or writer's certainty in } \\
\text { proposition }\end{array}$ & In fact/definitely/it is clear that \\
\hline Attitude markers & Express writer's attitude to proposition & $\begin{array}{l}\text { Unfortunately/I agree/ } \\
\text { surprisingly }\end{array}$ \\
\hline $\begin{array}{l}\text { Engagement } \\
\text { markers }\end{array}$ & $\begin{array}{l}\text { Explicitly refer to or build relationship } \\
\text { with reader }\end{array}$ & $\begin{array}{l}\text { Consider/note that/you } \\
\text { can see that }\end{array}$ \\
\hline Self-mentions & Explicit reference to author(s) & I/we/my/our \\
\hline
\end{tabular}

denominaba Interactional resources. Basándose en los estudios de Biber y Finegan (1989), Hunston (1994) y Labov (1984), introduce los conceptos de stance y engagement. El primero de ellos se correspondería con la imagen que el autor proyecta de sí mismo en su discurso. El segundo centra su atención en reconocer la presencia del futuro lector y, consecuentemente, establecer una interacción satisfactoria (Hyland, 2008). Con estos dos fenómenos incluidos en su taxonomía para el análisis del metadiscurso, Hyland no solo reparte los grupos que ya había presentado, sino que actualiza uno de ellos y, además, introduce cinco nuevos.

En el grupo de stance se mantienen hedges, boosters, attitude markers y self mention, que explicaremos brevemente:

- Hedges y boosters se corresponden con las estrategias pragmáticas que en español conocemos como atenuación e intensificación (ambos explicados en el apartado 2.2).

- Attitude markers: son expresiones mediante las cuales el autor expresa alguna opinión o sentimiento con respecto al enunciado al que se aplica. 
- Self mention: automenciones con las que el autor se hace presente en el discurso.

Desaparece el grupo denominado engagement markers, que pasa, evidentemente, a la rama de engagement. Esta rama se estructura de la siguiente manera:

- Reader mention: menciones explícitas de los lectores en el discurso científico.

- Questions: enunciados interrogativos con los que se finge un diálogo con el futuro lector.

- Shared knowledge: generalizaciones con las que se pretende que la comunidad científica acepte la información que presenta como saber compartido.

- Directives: con un nuevo término, Hyland (2008) modifica también la definición y pasan de ser referencias con las que crear un vínculo a expresiones con las que ordenar que el lector realice alguna actividad: como ver una figura que acompaña el texto o aceptar una clasificación propuesta por el autor.

\subsection{Un modelo de metadiscurso en español}

En el ámbito hispánico podemos encontrar estudios sobre el discurso científico como Oliver del Olmo (2004), Montolío (2014); Morales, Cassany y González (2008); Morales y Cassany (2008); Regueiro y Sáez (2013), entre muchos otros. Pese a los grandes avances que se han hecho entorno a los géneros académico y científico, los estudios sobre el metadiscurso en lengua española son escasos, entre los que podemos destacar Beke (2005), Suau Jiménez (2010) o Salas Valdebenito (2015); y en su gran mayoría aceptan el modelo propuesto por Hyland para el español. Sin embargo, tras un análisis profundo de varios textos hemos podido comprobar que el modelo desarrollado para el inglés no se puede aplicar en su totalidad al español. Por ello, hemos propuesto la siguiente taxonomía para el metadiscurso interpersonal:

A continuación, expondremos brevemente cada una de las categorías que podemos observar en la Tabla 2.

\subsubsection{Atenuación e intensificación}

Mientras la atenuación suele definirse como una estrategia o categoría pragmática que permite al hablante disminuir el efecto de lo dicho o del decir (Albelda, 2013; Briz, 1995, 1998), la intensificación se utiliza para lo contrario, esto es, destacarlo o remarcarlo (Albelda, 2002). Incluimos en estos apartados, por tanto, todos los elementos del discurso científico que permiten al autor bien minimizar su presencia en el texto o la fuerza de sus aseveraciones, bien acentuarla. Como muchos 
Tabla 2: El metadiscurso en español (Fuente: Chao Parapar, 2018).

ATENUADORES

INTENSIFICADORES

EVALUADORES

MARCADORES DE INTERACCIÓN

MARCADORES PERSONALES

REFERENCIALES
"Esto podría deberse a que la salud de los pacientes con más edad se encuentra muy deteriorada, haciéndolos no aptos para un trasplante" (Medicina)

"Evidentemente, también han entrado palabras del inglés que en su original contienen el sonido palatal sordo y que no necesariamente entran por la emigración" (Lingüística y Literatura)

"Es difícil saber el porcentaje de pacientes que sufre un duelo complicado ante la psicosis" (Psicología y Psiquiatría)

Directrices Textuales “... puede ser visto como una realización de una estructura jerárquica (vea Figura 1)" (Matemáticas)

Físicas “... luego se toma una alícuota de $50 \mathrm{~mL}$ y [se] traslada a un balón de $100 \mathrm{~mL} . . . "$ (Ingeniería)

Cognitivas “... hay que resaltar la importancia de implementar una insensibilización perfecta..." (Zoología y Veterinaria)

Acercamientos Apelaciones “... permítaseme traer a colación varios casos en los cuales" (Derecho)

$\begin{array}{ll}\text { Plural } & \text { "En los últimos años hemos asistido a } \\ \text { inclusivo } & \text { una nueva edición de las «guerras edu- }\end{array}$ cativas»..." (Derecho)

Preguntas “qué poesía no es "palabra esencial” $o$ no se ocupa de la "esencia" de las cosas?" (Lingüística y Literatura)

Apartes Informativas “... se constituyeron en lugar de resistencia (pasiva) de una población abocada al abismo ..." (Historia y Estudios Culturales)

De opinión “(quizá por eso Lorca se refería a la masturbación como "el sacrificio del semen"...)" (Arte)

De autor o automenciones "Me parece conveniente partir de una definición de lo que entendemos por cultura..." (Ciencias Económicas)

De lector "Tenéis (tenemos) el honor y el placer de escribir en un folio en blanco..."

(Educación)

“Algunos autores afirman que las mujeres con cáncer de mama más jóvenes padecen a menudo distintos efectos secundarios que las mayores y enfrentan diferentes desafíos que las pacientes posmenopáusicas, como esterilidad, pérdida de la masa ósea, menopausia prematura y mayor fatiga" (Medicina) 
otros trabajos, entendemos estas categorías como fenómenos escalares (Caffi, 1999), lo que en nuestro caso permite, además, que algunos elementos cumplan varias funciones metadiscursivas al mismo tiempo.

Lo que podría indicar que, a lo mejor, los hablantes no produjeron los enunciados iniciales como mandatos sino más bien como solicitudes o peticiones. (Lingüística y Literatura)

En el desarrollo de los estudios por muestreo, es de suma importancia la escogencia de un adecuado diseño muestra. Es decir, una forma correcta de obtener información vital, junto a un estimador que refleje de manera aproximada, al expandir la muestra, un resultado global para la población y muestre así el comportamiento de la misma. (Matemáticas)

Las expresiones que hemos destacado en cursiva en los ejemplos son casos de atenuación e intensificación, respectivamente. En (1) el uso del verbo poder, y en su forma condicional, reduce la intensidad de la afirmación que se va a realizar, esto es, que los enunciados objeto de estudio no son en realidad de la naturaleza que se pensaba al comienzo del estudio. Asimismo, el autor ve la necesidad de asegurar su atenuación y utiliza la expresión a lo mejor, que, según el Diccionario de partículas discursivas del español (Padilla García, 2008), la atenuación se produce porque quien lo utiliza no se hace responsable de lo que dice.

Por su parte, los segmentos que se han destacado en (2) es un claro caso de intensificación. La estructura de suma importancia se aplica a un aspecto de metodología del estudio, la acción de recogido de muestras para su posterior análisis. Con la expresión intensificadora el autor destaca dos cosas en especial: la delicadeza y el cuidado que ha de suponer elegir los objetos de estudio, así como el efecto que esta selección va a tener en las conclusiones que derivarán de su análisis. Algo parecido ocurre con el uso del adjetivo vital, que se aplica al sustantivo información, puesto que se trata de una reformulación del enunciado anterior en la que se focaliza la atención ya no el proceso de muestreo, sino en los datos que se van a obtener de dicho método.

\subsubsection{Los evaluadores}

El grupo de los evaluadores es uno de los más amplios, puesto que puede comprender desde adverbios (lamentablemente) hasta enunciados complejos ([años] en los que la firma del anciano polemista apenas asoma). ${ }^{1}$ En él incluimos todas las expresiones que, de algún modo, describen el contenido del texto desde la

1 Ambos ejemplos se encuentran en el corpus de Lingüística y Literatura. 
perspectiva del autor. Es habitual que cumplan al mismo tiempo la función de atenuación o intensificación.

(3) Dado que la recuperación física en absoluto tiene lugar y que, no obstante, el poema sigue relatando las hazañas de los guerreros, no parece descabellado asimilar el significado de Helena a la conducta heroica, más cuando para la imaginación de algunos griegos, como la de Eurípides en la obra sobre el mismo personaje, la adúltera en ningún momento se trasladó a Troya. (Lingüística y Literatura)

En el ejemplo destacamos un caso de función múltiple. En primer lugar, nos encontramos con una directriz cognitiva, concepto que se explicará en los siguientes párrafos, parecer + adjetivo + infinitivo. Mediante el uso de estos elementos, el autor indica al lector cómo debe proceder ante cierta información, en este caso, cómo debe interpretar el significado de un poema. En segundo lugar, tenemos una evaluación, estrechamente ligada a esta estructura por el uso del adjetivo que se introduce entre ambos verbos. En el ejemplo se utiliza descabellado, esto es, la directriz y la evaluación se suman para que el autor nos pueda convencer de que su interpretación de un poema es plausible. Por último, el verbo parecer atenúa la directriz en la que se inserta, por lo que consigue reducir la fuerza de la obligación.

\subsubsection{Los marcadores de interacción}

Dentro de este grupo, entran los elementos que explicitan una interacción entre los interlocutores del discurso científico. Estos se dividen, a su vez, en tres categorías: directrices, acercamientos y apartes.

- Directrices: órdenes que el autor da al lector del texto. Se diferencian tres subgrupos dependiendo del tipo de orden que se le dé: textuales, si lo redirige a otras partes del texto -apartados, párrafos, tablas o, como podemos ver en (4), figuras-; físicas, si se indica qué debe hacer fuera del discurso; cognitivas, si le pide al lector que realice una acción mentalmente.

- Acercamientos: son expresiones que rompen la distancia preestablecida para incluir al lector y, aprovechando la cercanía, persuadirlo con mayor facilidad.

(4) ...y cuyos materiales arenosos se encuentran empotrados en el tope arcilloso de los abanicos aluviales Q2 mejor consolidados del Pleistoceno medio y de los depósitos lacustres Q1, propiciando de esta manera la ubicación en los mismos de mesas locales de agua emperchada (Singer et al. 2007, véase figura 20). 
Permítaseme, para ejemplificar la hipótesis anterior, analizar el modo en que una comedia urbana del primer Lope, La viuda valenciana, propone representaciones de algunos aspectos concretos de la subjetividad barroca.

Como podemos observar, el párrafo -que inicia un apartado- comienza con una petición o ruego al lector. Aun sabiendo que no va a recibir el permiso que pide, el autor gana confianza con su público al hacerlo partícipe de su discurso. Por eso lo consideramos un acercamiento al lector, esto es, una ruptura con la distancia que se presupone en el discurso académico-científico. Puede ser esta la causa por la que considere necesario el uso de métodos de atenuación. Uno de ellos sería la conjugación del verbo (que no es permíteme, permítame o permítanme), pero el que más llama la atención es el segmento que hemos señalado en cursiva (para ejemplificar...), que adelanta la justificación incluso antes de indicarnos cuál es la petición en cuestión.

- Apartes: en este grupo se incluye todo el contenido que se aporta de forma secundaria (mediante el uso de guiones, paréntesis, corchetes...), ya sea información adicional a la que se proporciona en el cuerpo, como vemos en el siguiente ejemplo, ya sean opiniones del propio autor.

(6) La expresión guardar un minuto de silencio (apareció entre las repuestas de los encuestados, sin embargo, no forma parte del análisis) es muy productiva en el español de México. Si bien es cierto que esta frase no focaliza el término de un proceso (la muerte), sugiere la noción o la idea de que puede ser utilizada para referirse a morir, aunque su verdadero significado esté vinculado con una expresión de luto que simboliza la pérdida de alguien, o bien, la conmemoración de un suceso trágico. (Lingüistica y Literatura)

\subsubsection{Los marcadores personales}

Son menciones explícitas del autor y del lector. Pese a los debates que pueden encontrarse en múltiples manuales o guías de estilo en torno a la presencia de los interlocutores en el discurso científico, nuestro corpus refleja una clara presencia del autor y, hasta el momento, muy sutiles menciones al lector:

(7) En estos términos, el autor concluye que 'si C y E son dos sucesos aleatorios y $\mathrm{C}$ causa a $\mathrm{E}$, entonces $\mathrm{P}(\mathrm{E} \mid \mathrm{C}) \leq \mathrm{P}(\mathrm{E})$ ”. Es aquí en donde creo que el susodicho autor debería haber razonado un poco más acerca de cuándo se tiene la igualdad en esta regla de probabilidades...

(Matemáticas) 


\subsubsection{Los referenciales}

Son expresiones vagas que intentan dar la impresión de que lo que se expone en el texto está aceptado por la comunidad científica, pero sin aportar referencias bibliográficas que lo demuestren. Veamos:

$$
\begin{aligned}
& \text { El bagaje teórico y analítico acumulado en estas décadas ha elevado } \\
& \text { enormemente nuestro grado de comprensión acerca de los mecanismos } \\
& \text { que rigen la interacción social entre los individuos, de la que -es bien } \\
& \text { sabido- la interacción lingüística resulta un componente esencial. }
\end{aligned}
$$

Podemos observar en el ejemplo que, mediante el uso de un aparte, se introduce la expresión que pretende disipar toda duda sobre si la interacción lingüística es o no un componente tan importante en la interacción social.

\section{Marco metodológico}

Para la elaboración del estudio de los marcadores discursivos en cualquier caso, en todo caso, de todos modos, de todas formas y de todas maneras, se ha utilizado un enfoque basado en la lingüística de corpus. Han sido necesarias tres fases: en primer lugar, se han obtenido las ocurrencias de dichas expresiones en el corpus utilizando el programa informático AntConc (Anthony, 2018); en segundo lugar, se han estudiado cada uno de los contextos en los que se hallaban para determinar cuáles debían incluirse y cuáles excluirse; ${ }^{2}$ finalmente, se ha calculado la frecuencia de aparición de los cinco marcadores.

Hemos partido de un amplio corpus compuesto por quince disciplinas científicas, de entre las cuales hemos seleccionado cuatro: Educación, Ingeniería, Matemáticas y Lingüística y Literatura. ${ }^{3}$ De este modo, cada una de ellas actúa como representante de una de las áreas del conocimiento (Ciencias Sociales, Ciencias Aplicadas, Ciencias y Artes y Humanidades, respectivamente ${ }^{4}$ ).

2 Para discriminar los casos se utilizaron los tres principios clave que Hyland y Tse (2004) establecen para el metadiscurso.

3 Una de las medidas adoptadas en el momento de la creación del corpus fue la de crear un único subcorpus con las dos disciplinas: cincuenta textos de Lingüística y cincuenta de Literatura.

4 La nomenclatura empleada en este trabajo pertenece a la clasificación que presentaba la base de datos a partir de la cual hemos creado nuestro corpus. No obstante, dicha web, la Red Iberoamericana de Innovación y Conocimiento Científico, cambió a comienzos de 2018 la clasificación aquí utilizada por una nueva en la que se presentan cinco ramas: Ciencias de la Salud, Ciencias de la Vida, Ciencias Físicas e Ingeniería, Ciencias Sociales y Humanidades, Multidisciplinar. Clasificación que adoptaremos en futuros trabajos. 
Los subcorpus contienen cien textos escritos en español, lo que quiere decir que en este estudio se han examinado cuatrocientos artículos de investigación científica. Todos cumplen con los siguientes criterios para garantizar tertium comparationis ${ }^{5}$ (Biber, 2004; Connor, 2004), es decir, una similitud entre los textos que nos permita equipararlos:

1. Estar indexados en la web Red Iberoamericana de Innovación y Conocimiento Científico (REDIB)^: https://www.redib.org/?lng=es

2. Estar disponibles en Portable Document Format (PDF).

3. Ser textos originales en español, es decir, no se han admitido traducciones de artículos científicos de otros idiomas.

4. Ser artículos de investigación empírica, es decir, se han excluido estudios de caso, revisiones de la literatura, ensayos...

5. Tener una extensión máxima de 30 páginas. $^{6}$

6. Haber sido publicados entre enero de 2010 y febrero de 2018.

En lo referente a las características internas del corpus, los textos seleccionados se han estudiado teniendo en cuenta la integridad del género discursivo al que pertenece, esto es, solo se han excluido los títulos, palabras clave y resúmenes en inglés (abstract), si los hubiere, así como los apartados de referencias bibliográficas. En cuanto al contenido de tablas, figuras y gráficas, aunque sí se han mantenido sus títulos o pies aclaratorios, todo el texto que pudieran contener ha sido descartado. Por este motivo, hemos igualado los subcorpus, como se ha mencionado con anterioridad, por número de textos y no por números de palabras. De este modo, la comparación entre ciencias se realiza manteniendo las características propias de los discursos de cada comunidad discursiva, incluyendo la estructura y la extensión de los textos.

5 El concepto de tertium comparationeis se aplica en los estudios que emplean corpus comparativos. Aunque nuestro corpus no se ha construido con esas características, sí consideramos oportuno que los subcorpus que lo componen cumpliesen con los requisitos necesarios para garantizar la comparabilidad.

6 La extensión de los artículos que forman el corpus tiene un mínimo de 10 páginas y un máximo de 30 en las consideradas "ciencias blandas" y un mínimo de 7 páginas para las "ciencias duras". Con el máximo podíamos hacer algunas excepciones si, como ocurría en algunos textos de Derecho, las notas a pie de páginas ocupaban media página o más. Como puede suponerse, los artículos de investigación de las "ciencias duras" no son tan extensos como para tener que imponer un límite. 


\section{Estudio de los marcadores}

\subsection{El marcador en cualquier caso}

En su capítulo de la Gramática descriptiva de la lengua española, Martín Zorraquino y Portolés Lázaro (1999) consideran este marcador un reformulador de distanciamiento. Esta clase de reformuladores no presenta un miembro anterior en el discurso formulado de manera diferente, sino que lo muestran como poco relevante, para luego introducir un nuevo miembro importante para la prosecución del discurso (Garcés Gómez; 2014; Martín Zorraquino y Portolés Lázaro, 1999; Montolío, 2014, Portolés, 2008). No obstante, la clasificación de en cualquier caso entre los reformuladores no es unánime en la bibliografía y lo encontramos también entre los conectores contraargumentativos o, más concretamente, entre los concesivos (cfr. Fuentes Rodríguez y Alcaide Lara, 2002; Nueva gramática de la lengua española, 2009).

Por nuestra parte, a la hora de explicar el funcionamiento de este y de los restantes marcadores del presente estudio, nos vamos a decantar por su consideración de reformuladores de distanciamiento, ya que, como comprobaremos, esta adscripción se ajusta al funcionamiento de dichas unidades en los textos estudiados. Así, utilizando este marcador el autor obliga al lector a realizar una revisión de las diferentes opciones que ha expuesto previamente e introduce en el nuevo miembro del discurso un argumento o punto de vista incuestionable o definitivo (Garcés Gómez, 2014; Portolés, 1998, 2008). De este modo, como indica Montolío (2014), el autor solo se compromete con el contenido del segundo miembro.

$$
\begin{aligned}
& \text { En general, las tasas de participación de los padres es menor en } \\
& \text { poblaciones históricamente desfavorecidas, dando lugar a los } \\
& \text { argumentos acerca de que facilitar la participación en estas poblaciones } \\
& \text { puede reducir las brechas de logro (Crosnoe y Huston, 2007). Sin } \\
& \text { embargo, también se encuentra que no siempre son los padres de } \\
& \text { contextos socioculturales más bajos los que prestan menor atención a } \\
& \text { sus hijos. Se dan casos de padres muy preparados y que ejercen } \\
& \text { profesiones de alta cualificación muy ocupados que dedican muy poco } \\
& \text { tiempo a sus hijos (Pérez Serrano, 1984). En cualquier caso, cuando se } \\
& \text { aborda el tema desde un enfoque sistémico, se describe la relación } \\
& \text { familia y escuela como compensatoria. [sic] (Educación) }
\end{aligned}
$$

En el ejemplo podemos observar una construcción característica: se introducen dos o más opciones, teorías o experiencias que serán refutadas o cuestionadas 
para favorecer la infalibilidad de la conclusión del autor. En el fragmento anterior se presentan dos tipos de referentes paternos muy diferentes entre ellos; sin embargo, parece ser que desde el enfoque que sigue el autor o el que considera más oportuno, el tipo de familia en el que se enmarca la vida del alumnado no influye en la relación de estos con la escuela.

En muchos casos, como indican Portolés (1998) y Garcés Gómez (2014), la disyuntiva previa al uso del marcador no se hace explícitamente, sino que el interlocutor debe encontrarla en la complejidad del primer miembro. Asimismo, en el lenguaje de la ciencia puede presentarse la disyuntiva en la interpretación de un elemento extratextual, al que los autores hacen referencia mediante el uso de una directriz textual (Chao Parapar, 2018; Hyland, 2010).

En la Tabla 11 se observa que, en cualquier caso, la eficiencia está muy cercana a uno, lo que nos indica que el algoritmo propuesto genera resultados satisfactorios y de no ser así, éste da la oportunidad de dirigir la búsqueda hacia nuevas regiones mediante la diversificación o mediante un permutación inicial [sic] que no sea la natural.

(Matemáticas)

En estos casos el lector debe recurrir a la tabla, figura o gráfica e interpretarla. Una vez analizados los datos, el propio lector debe imaginar las diversas opciones que estos le proporcionan. Cuando ya ha realizado todo el proceso mentalmente, está preparado para comprender el uso del marcador por parte del emisor. Sin embargo, todas las conclusiones a las que haya podido llegar son insignificantes para la argumentación que se presenta en el segundo miembro del discurso. Así, en el ejemplo (10) el autor del artículo indica que sean los resultados menores o mayores a uno, su cercanía a este número prueba que el algoritmo del que se habla es válido o que, en caso de no serlo, sigue siendo útil, ya que ayuda a obtener el resultado buscado.

Aunque en lenguaje coloquial es habitual encontrar el uso de en cualquier caso con una oración negativa que descarte los puntos de vista de uno de los participantes (Garcés Gómez, 2014), nuestros resultados muestran que no es común en el lenguaje científico negar la oración en la que se utiliza este marcador. El único caso que se ha obtenido en el corpus es el que sigue:

Así, ella [Luisa], como muchos de los habitantes de Aduero, lo ve en su faceta de Guzmán, mientras que Gráez y unos pocos más, como la Princesita, le defienden como Aurelio. No es, en cualquier caso, Luisa la única que favorece el desmentido de Aurelio. Los hechos mismos lo propician, como cuando aparece muerto un mendigo que se había imaginado como un Fausto. (Lingüística y Literatura) 
Como podemos ver en el ejemplo, el marcador se ha insertado en una oración copulativa enfática, con lo que el autor consigue un mayor efecto de contraste entre los dos miembros de discurso. Por un lado, tenemos el miembro sobre el que recae la concesión (el enfrentamiento entre vecinos y el tratamiento a cierto personaje de la novela que se estudia); por otro, el argumento del autor que ya no se sitúa en otro plano de la argumentación solo con el marcador discursivo, sino también al resaltar el segundo miembro con la oración copulativa enfática (Luisa no es la única que...).

En este caso no podemos concluir que el uso de la negativa sea para contraponer su punto de vista al de otro participante (en este contexto, el lector). Probablemente no podamos encontrar ejemplos de ese uso en nuestro corpus debido al tipo de género con el que estamos trabajando, ya que el autor debe acercarse al lector con mesura.

Normalizados los resultados obtenidos, la frecuencia de aparición de en cualquier caso en el corpus es de 2,15 por cada 100000 palabras. Como podemos observar en la Gráfica 1, la disciplina científica en la que se ha obtenido un mayor porcentaje ha sido Lingüística y Literatura, algo esperado, puesto que el discurso de las Humanidades suele establecer más contacto con el lector que en los discursos de las ciencias. Es llamativo, sin embargo, que Educación presente solo un 6,98\%, lo que contradice la hipótesis de la que se partía en este estudio: el uso será mayor en lo que se conoce como “ciencias blandas”, es decir, Ciencias Sociales y Artes y Humanidades. Matemáticas nos sorprende no por su casi $10 \%$, sino por haber superado a Educación. No obstante, Ingeniería ha ofrecido los datos esperados.

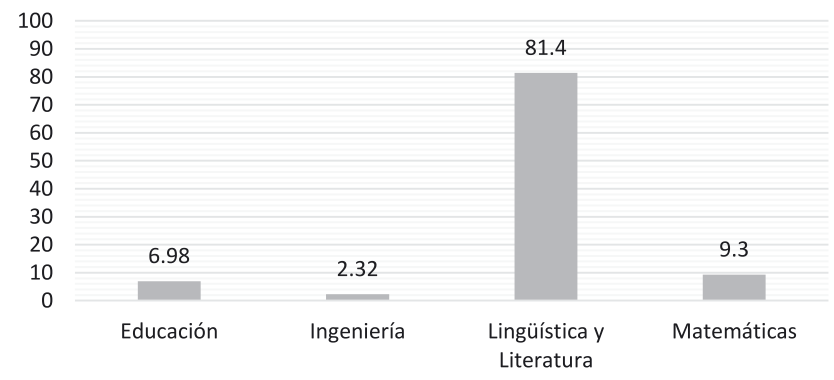

Gráfica 1: Comparación del uso de en cualquier caso 


\subsection{El marcador en todo caso}

Como en cualquier caso, nos encontramos ante un reformulador de distanciamiento (Garcés Gómez, 2013; Martín Zorraquino y Portolés Lázaro, 1999; Montolío, 2014; Portolés, 2008), considerado también concesivo por otros autores (Fuentes Rodríguez y Alcaide Lara, 2002; Santos Río, 2003). Hay diferencias entre ambos marcadores, como señaló Portolés, para quien en todo caso no coincide con en cualquier caso, ya que "vincula en sus usos específicos dos miembros del discurso de tal forma que el segundo invalida la pertinencia del primero, pero reemplazándolo" (1998: 257).

El volumen del corpus, adecuado para un trabajo de estas características, no nos ha permitido extraer unos resultados concluyentes en cuanto a la relación entre la atenuación y las diferentes ramas de conocimiento; en todo caso, es posible afirmar una presencia menor de atenuación en las tesis de Ciencias y de Ciencias de la Salud, que son también aquellas en las que el capítulo de "Conclusiones" es notoriamente más breve (cfr. nota 10). (Lingüística y Literatura)

En este ejemplo, podemos observar que el autor del texto presenta en el primer miembro de su discurso un problema o impedimento que ha surgido durante la realización de su estudio: el volumen del corpus no le ha permitido extraer resultados concluyentes. En el segundo miembro, expone que, a pesar de las dificultades encontradas en el camino, ha sido capaz de llegar a una conclusión: la atenuación está menos presente en las tesis de las ramas de Ciencias y Ciencias de la Salud.

Sí hay, empero, contextos en los que su significado es equivalente al de en cualquier caso, como vemos en

García-Miguel asigna un significado único y constante a cada preposición, normalmente vinculado al ámbito local, que se destaca como el más frecuente o prototípico. Dicho significado podría derivar hacia sentidos más abstractos o nocionales en virtud de las características significativas del predicado, pero en todo caso serían compatibles y más o menos próximos a ese significado prototípico. (Lingüística y Literatura)

En este fragmento se presentan dos tipos de significado de las preposiciones utilizadas con varios predicados: unos más abstractos, otros más nocionales. A pesar de haberlas dejado reflejadas explícitamente, con el uso del marcador descarta la importancia de esas diferencias para indicar que, sea cual sea el significado de cada una de las preposiciones, seguirán siendo compatibles o cercanos al 
significado prototípico. Por ello, podríamos reformular el fragmento con en cualquier caso sin que el significado del contenido proposicional se vea afectado:

Dicho significado podría derivar hacia sentidos más abstractos o nocionales en virtud de las características significativas del predicado; pero, en cualquier caso, serían compatibles y más o menos próximos a ese significado prototípico.

Asimismo, Fuentes Rodríguez y Alcaide Lara (2002) indican que este marcador tiene también un uso del que carece el marcador estudiado en el apartado anterior, ya que puede emplearse como operador discursivo restrictivo. Para García Negroni (2002) este valor tendría también la capacidad de minorar la fuerza argumentativa, es decir, posee un claro valor atenuante.

$$
\begin{aligned}
& \text { Estas oraciones son agentivas, activas, que mucho difieren de las } \\
& \text { referidas antes, pues no estamos frente a un agente (ya que este no } \\
& \text { puede ser argumento de un verbo de estado), sino, en todo caso, frente a } \\
& \text { un «experimentador», quien «experimenta» en sí el estado o sensación } \\
& \text { que nombra el verbo, y frente a un «estímulo», que es la causa del } \\
& \text { desencadenamiento del «estado» en cuestión (en este caso, el estado } \\
& \text { de «gustar»). (Lingüística y Literatura) }
\end{aligned}
$$

En el segmento anterior el emisor se debate entre los diferentes tipos de oraciones sobre las que hace el estudio. De entre todos los complementos, descarta el que considera erróneo (agente) y presenta la opción que le parece correcta (experimentador/estímulo). Con el marcador lo que hace es restringir la validez del primer miembro del discurso al tiempo que introduce el argumento que considera más probable. Fuentes Rodríguez y Alcaide Lara (2002) indican que, a su vez, tiene un valor argumentativo y modal de hipótesis, con la posibilidad de combinarlo con sino, conjunción que, además, podemos ver en el ejemplo (14). Es en estos casos en los que se percibe la función atenuante, la cual entendemos como una estrategia pragmática con la que se pretende minimizar la fuerza ilocutiva de los actos de habla y que, por lo tanto, reduce el efecto de lo dicho o lo hecho (Albelda y Cestero, 2011; Briz, 1995; Briz y Albelda, 2013; Caffi, 1999, 2007). En este caso, el autor se distancia de su texto e introduce el segundo miembro como una posibilidad con la que no se compromete.

Normalizados los resultados obtenidos, la frecuencia de en todo caso en el corpus es de 2,75 por cada 100000 palabras. En la Gráfica 2 podemos ver que la disciplina que presenta un mayor uso del marcador es, de nuevo, Lingüística y Literatura. En este caso, vuelven a sorprendernos los datos obtenidos en el subcorpus de Educación que manifiesta el mismo porcentaje de uso que el de Ingeniería. Al contrario de lo que ocurría con el marcador anterior, Matemáticas no 


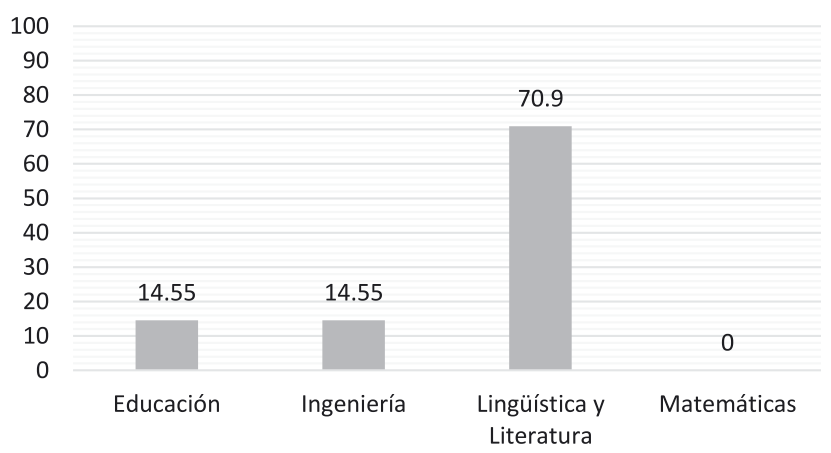

Gráfica 2: Comparación del uso de en todo caso.

presenta ni un solo caso en el que se utilice en todo caso, lo que cabe dentro de lo esperado por una disciplina de Ciencias.

\subsection{Los marcadores de todos modos, de todas formas y de todas maneras}

Como los marcadores estudiados en los apartados anteriores, en esta sección se estudian tres reformuladores de distanciamiento que "presentan expresamente como no relevante para la prosecución del discurso un miembro [...] anterior" (Portolés, 1998:142). Así, como ocurría con en cualquier caso, en los artículos de investigación científica, se utilizan para restar importancia a los datos o teorías que se han expuesto y presentan, en el segundo miembro, la información que el autor prefiere que el lector considere más importante.

(15) Ahora bien, no siempre se muestra con claridad en el titular el discurso ajeno, aunque puede estar presente en el texto. De todos modos, hay que partir de la idea de que cualquier texto es polifónico, de acuerdo con el pensamiento de Ducrot (1984, p.205) ... (Lingüística y Literatura)

(16) La selección de unos valores y no de otros es siempre arriesgada y, más todavía, cuando incide en un elemento tan trascendental como la educación. Escoger es renunciar y renunciar es siempre comprometido. De todas maneras, según manifiestan Pujol y Luz (2003), los valores humanos se entrelazan entre ellos y resulta difícil distinguir dónde termina uno y dónde empieza otro. (Educación) 

Por lo pronto, la solución sería cambiar el tipo de dato int a tipo de dato long (que son enteros que se almacenan en 4 bytes) y de esa forma se amplía el rango de números a evaluar. De todas formas, a menos que se utilicen simulaciones a partir de estructuras de datos y mientras este tipo de ejercicios se fundamenten en los tipos de datos primitivos, siempre existirá una cota superior que limita el número a evaluar. (Matemáticas)

Santos Río (2003) clasifica al marcador de todos modos como una locución adverbial no-hipotética con el significado de 'comoquiera que sea' o una locución adverbial concesiva no-hipotética equivalente a 'a pesar del hecho o conjunto de hechos expuestos'. En los ejemplos anteriores nos encontramos con el segundo tipo de locución, la concesiva. Independientemente de la forma, manera o modo previos al segundo miembro, la continuación del discurso dependerá de la conclusión expuesta. Si volvemos al ejemplo (15) vemos que, aunque el texto no refleje claramente el discurso de otros emisores, siempre debemos tener en cuenta que todos los textos son polifónicos.

Como ya indicaban Martín Zorraquino y Portolés (1999), estos marcadores son menos frecuentes que los estudiados en los apartados anteriores, y así lo reflejan los resultados de nuestro análisis. Tras normalizar dichos resultados, la frecuencia total del uso de los tres marcadores es de 1,75 por cada 100000 palabras: de todos modos con 0,90, de todas maneras con 0,30 y de todas formas con 0,55.

En la Gráfica 3 podemos observar que la disciplina científica en la que se ha obtenido un mayor porcentaje ha sido Lingüística y Literatura. En esta no solo destaca el uso de de todos modos, sino que se han obtenido los resultados más altos de los tres marcados. Resultan interesantes, de nuevo, los datos correspondientes a Educación que, pese a ser la segunda ciencia con mayor porcentaje de uso, está mucho más cerca de la escasa representación propia de Ingeniería y Matemáticas que del frecuente uso en Lingüística y Literatura. No obstante, Matemáticas e Ingeniería han aportado los datos esperados: poca frecuencia, en caso de haberla.

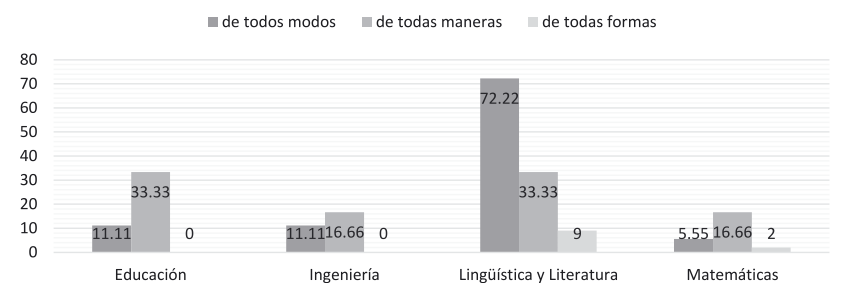

Gráfica 3: Comparación, de todos modos, de todas formas y de todas maneras. 
Tabla 3: Frecuencia de posiciones en el discurso.

\begin{tabular}{lrrr}
\hline & Posición inicial & Posición media & Posición final \\
\hline en cualquier caso & 22 & 21 & 0 \\
en todo caso & 25 & 30 & 0 \\
de todas formas & 8 & 3 & 0 \\
de todas maneras & 3 & 3 & 0 \\
de todos modos & 15 & 4 & 0 \\
N.o concordancias & 73 & 61 & $\mathbf{0}$ \\
Porcentajes & $54 \%$ & $46 \%$ & $\mathbf{0} \%$ \\
\hline
\end{tabular}

\subsection{Generalidades: posición en el discurso}

La bibliografía parece concordar en que los cinco marcadores pueden aparecer en las tres posiciones respecto al miembro discursivo en que comparecen: inicial, media o final (Fuentes Rodríguez, 1995-1996; Fuentes Rodríguez y Alcaide Lara, 2002; Martín Zorraquino y Portolés, 1999; Ruiz Garrillo, 2008; Santos Río, 2003). Sin embargo, los datos obtenidos del corpus indican que en el discurso científico no se utilizan en posición final (Tabla 3).

Es probable que esta ausencia de concordancias de marcadores en posición final se deba al registro utilizado en los artículos de investigación empírica. Debido al contexto en el que se sitúa la comunicación, los argumentos deben presentarse de forma clara, objetiva y ordenada (Castelló, 2007; Montolío, 2014). Por ello, la posición del marcador parece ser mucho más recomendable al inicio, ya que de este modo el lector comprende desde el principio el razonamiento del autor.

En muchos casos la extensión del segundo miembro requiere que el marcador aparezca bien al comienzo, bien en posición media. Lo mismo ocurre cuando el autor aporta aclaraciones (ejemplo 18) o concatena una serie de argumentos (ejemplo 19) porque el significado del marcador no se aplicaría al miembro que contiene el contenido proposicional requerido para la continuación del discurso.

(18) En cualquier caso, el proceso inferencial realizado por el oyente supone recuperar la intención del hablante en contextos particulares, y en eso consiste el significado pragmático de la emisión. (Lingüística y Literatura)

(19) En todo caso, según la teoría de la transitividad que defendemos, estos verbos no serían considerados como intransitivos, pues observamos que la única construcción intransitiva es la construcción (41); mientras que en el resto aparecen argumentos transitivos, incluido el complemento 
de régimen preposicional, que en los siguientes ejemplos es doble... (Lingüística y Literatura)

Sí sería posible la reformulación de (18a), pero no la de (18b):

(18a) El proceso inferencial realizado por el oyente supone, en cualquier caso, recuperar la intención del hablante en contextos particulares, $\mathrm{y}$ en eso consiste el significado pragmático de la emisión. (Lingüística y Literatura)

El proceso inferencial realizado por el oyente supone recuperar la intención del hablante en contextos particulares, y en eso consiste el significado pragmático de la emisión, en cualquier caso. (Lingüística y Literatura)

\section{Conclusiones}

En el estudio que ahora concluimos hemos comparado la presencia de los marcadores del discurso en cualquier caso, en todo caso, de todos modos, de todas formas y de todas maneras en artículos de investigación pertenecientes a cuatro disciplinas científicas. Este trabajo nos ha permitido comprobar la rentabilidad de la metodología de la lingüística de corpus para análisis cuantitativos como el realizado, ya que se ha manejado una elevada cantidad de textos, y por ende de palabras, lo cual permite obtener resultados concluyentes, que serían difícil de alcanzar con otras metodologías.

En primer lugar, hemos de señalar que la comparación de concordancias contradice la hipótesis de que las "ciencias blandas" utilizarían dichos marcadores en mayor cantidad (ver Gráfica 4), pues el representante de las Ciencias Sociales (Educación) no muestra el uso esperado. Sin embargo, sí se han obtenido los

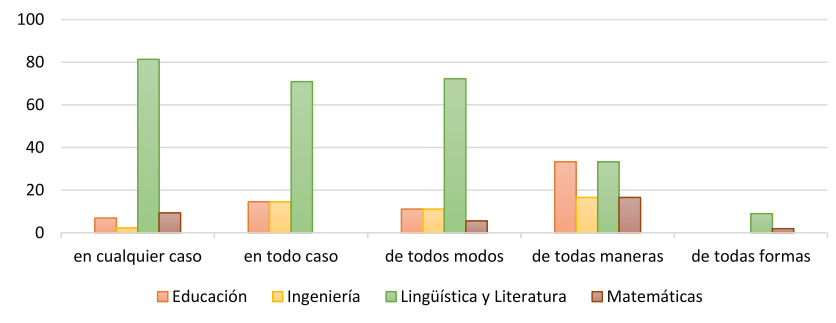

Gráfica 4: Comparación de los cinco marcadores. 
resultados esperados en Ciencias (Matemáticas) y Ciencias Aplicadas (Ingeniería). De todos modos, aunque la cantidad de textos examinados es representativa para un estudio de estas características, tan solo se ha analizado una especialidad por rama de conocimiento. Por consiguiente, podemos establecer conclusiones sobre las disciplinas estudiadas, pero no parece posible extenderlas a la rama científica que representan.

En segundo lugar, el género discursivo es diferente en estructura, extensión y, por supuesto, en el uso de la lengua. Parece ser que los artículos de Lingüística y Literatura tienden a centrarse en el hilo argumentativo, justificando el porqué de todo lo que allí se expone con teorías de las que parten y metodologías que han ayudado al avance en el campo. No obstante, Matemáticas e Ingeniería parecen centrarse en los datos obtenidos en el estudio, lo que se refleja mediante un mayor uso de elementos extratextuales. De este modo, parece más plausible que Lingüística y Literatura tenga que realizar concesiones y utilizar el distanciamiento como método atenuador.

Finalmente, podemos concluir que los marcadores discursivos deben tener un hueco en una taxonomía metadiscursiva. Con este trabajo, hemos conseguido comprender la complejidad de introducirlos en la clasificación propuesta en la Tabla 2 (Chao Parapar, 2018), puesto que las funciones que los marcadores cumplen en el plano metadiscursivo son múltiples. Por ello, creemos que lo más acertado sería clasificarlos principalmente dentro de la taxonomía del metadiscurso textual, en la que todavía estamos trabajando, aceptando que, en ocasiones, pueden cumplir tanto la función textual como la interpersonal.

\section{Referencias}

Acín Villa, E. (2001). Algo más sobre como. Anuario de estudios filológicos, 24, 9-24.

Acín Villa, E. (2019). Apuntes sobre pero bueno. En R. González Ruiz, I. Olza Moreno y O. Loureda Lamas (coord.), Lengua, cultura, discurso: estudios ofrecidos al profesor Manuel Casado Velarde, Universidad de Navarra (EUNSA).

Albelda, M. (2013). La atenuación: tipos y estrategias. En J. R. Gómez Molina (coord.), El español de Valencia: estudio sociolingüístico, 315-343.

Albelda, M. y Cestero, A. M. (2011). De nuevo, sobre los procedimientos de atenuación lingüística. Español Actual, 96, 9-40.

Albleda, M. (2002). El estudio de la intensificación como categoría pragmática. Interlingüística, 13, 115-128.

Anthony, L. (2018). AntConc versión 3.5.7. Tokyo, Japón: Waseda University. Tomado de [Software]. http://www.laurenceanthony.net/software.

Beke, R. (2005). El metadiscurso interpersonal en artículos de investigación. Revista Signos, 38(57), 7-18. 
Biber, D. (2004). Representativeness in corpus design. En G. Sampson D. y McGarthy (eds.), Corpus Linguistics: Readings in a widening discipline (pp. 174-197). Londres: Continuum.

Biber, D. y Finegan, E. (1989). Styles of stance in English: lexical and grammatical marking of evidentiality and affect. Text, 9(1), 93-124.

Boyero Rodríguez, M. J. (2002). Los marcadores conversacionales que intervienen en el desarrollo del diálogo. Madrid: Publicaciones Universidad Pontificia de Salamanca.

Briz, A. (1993a). Los conectores pragmáticos en español coloquial (I): su papel argumentativo. Contextos, $\mathrm{XI} / 21-22,145-188$.

Briz, A. (1993b). Los conectores pragmáticos en español coloquial (II). Su papel metadiscursivo. Español Actual, 59, 39-56.

Briz, A. (1994). Hacia un análisis argumentativo de un texto coloquial. La incidencia de los conectores pragmáticos. Verba, 21, 369-399.

Briz, A. (1995). La atenuación en la conversación coloquial. Una categoría pragmática. En L. Cortés (ed.) El español coloquial: Actas del I Simposio sobre análisis del discurso oral (pp. 103-222). Almería: Universidad de Almería.

Briz, A. (1998). El español coloquial en la conversación: esbozo de pragmagramática. Barcelona: Ariel.

Briz, A. y Albelda, M. (2013). Una propuesta teórica y metodología para el análisis de la atenuación lingüística en español y portugués. La base de un proyecto común (ES.POR.ATENUACIÓN). Onomázein, 28, 288-319.

Caffi, C. (1999). On mitigation. Journal of Pragmatics, 31, 881-909.

Caffi, C. (2007). Mitigation. Oxford: Elsevier.

Casado Velarde, M. (1991). Los operadores discursivos es decir, esto es, o sea y a saber en español actual: valores de lengua y funciones textuales (Vol. XIII, pp. 87-116). LEA.

Castelló, M. (2007). Escribir y comunicarse en textos científicos y académicos. Conocimientos y estrategias. Barcelona: Graó.

Chao Parapar, M. (2018). Procedimientos de atenuación en los artículos de investigación científica: las directrices cognitivas en español. En A. García Ramón y M. A. Soler Bonafont (eds.) ELUA: Estudios de atenuación en el discurso (pp. 139-156), Anexo IV.

Connor, U. (2004). Intercultural rhetoric research: Beyond texts. Journal of English for Academic Purposes, 3, 291-304.

Cortés Rodríguez, L. (1991). Sobre conectores, expletivos y muletillas en el español hablado. Málaga. Editorial Librería Ágora.

Crismore, A., Markkanen, R., \& Steffensen, M. S. (1993). Metadiscourse in persuasive writing: a study of texts written by American and Finish university students. Written Communication, 10(1), 39-71.

Eisner L. y Montemayor-Borsinger A. (2014). Perspectivas pragmáticas y funcionales sobre conectores espaciales con valor contraargumentativo: lejos de en discursos académicos. En M. M. García Negroni (coord.), Marcadores del discurso: perspectivas y contrastes (pp. 351-362), Buenos Aires: Santiago Arcos.

Escandell Vidal, M. V. (2004). Fundamentos de semántica composicional. Barcelona: Ariel.

Fuentes Rodríguez, C. (1990). Algunos operadores de función fática. Habla de Sevilla y hablas americanas. Sociolingüística andaluza, 5, 137-170.

Fuentes Rodríguez, C. (1995-1996). El lexema 'caso' y su rendimiento en el ámbito de la conexión. Pragmalingüística, 3-4, 329-349.

Fuentes Rodríguez, C. (2009). Diccionario de conectores y operadores del español. Madrid: Arco/ Libros. 
Fuentes Rodríguez, C. y Alcaide Lara, E. R. (2002). Mecanismos lingüísticos de la persuasión. Madrid: Arco/Libros.

Garcés Gómez, M. P. (2008). La organización del discurso: marcadores de ordenación y de reformulación. Madrid: Iberoamericana.

Garcés Gómez, M. P. (2013). El proceso evolutivo de los marcadores en todo caso y en cualquier caso. En Emili Casanova y Cesáreo Calvo (eds.) Actas del XXVI Congreso Internacional de Lingüística y Filología Románica (pp. 449-460). Berlín, Boston: De Gruyter.

Garcés Gómez, M. P. (2014). Los marcadores discursivos de topicalización en los textos de la ciencia (siglos XVIII-XIX). En M. Bargalló y C. Garriga (eds.) "Llaneza”: estudios dedicados al profesor Juan Gutiérrez Cuadradro (pp. 47-60). Universidade da Coruña.

García Negroni, M. M. (2002). En todo caso: reformulación y cortesía. En Actas del II Congreso Nacional de ALED (1-13), La Plata, UNLP.

Harris, Z. S. (1970 [1959]). Linguistic transformations for information retrieval. En Z. S. Harris Papers in structural and transformational linguistics. Formal linguistics series. Springer, Dordrecht.

Hunston, S. (1994). Evaluation and organization in a sample of written academic discourse. En

M. Coulthard (ed.) Advances in written text analysis (pp. 191-218). Londres: Routledge.

Hyland, K. (1998). Persuasion and context: The pragmatics of academic discourse. Journal of Pragmatics, 30, 433-454.

Hyland, K. (2000). Disciplinary discourses: Social interactions in academic writing. Londres: Edward Arnold.

Hyland, K. (2005). Metadiscourse: Exploring interaction in writing. London: Longman.

Hyland, K. (2008). Persuation, interaction and the construction of knowlodge: Representing self and others in research writing. International Journal of English Studies, 8(2), 1-23.

Hyland, K. (2010). Metadiscourse: Mapping interactions in academic writing. Nordic Journal of English Studies, 9(2), 125-143.

Hyland, K. y Tse, P. (2004). Metadiscourse in academic writing: A reappraisal. Applied Linguistics, 25(2), 156-143.

Labov, W. (1984). Intensity. En D. Schiffrin (ed.), Meaning, form, and use in context: Linguistic applications (pp. 43-70). Washington, D.C.: Georgetown University Press.

Loureda, O. y Acín Villa, E. (2010). Los estudios sobre marcadores del discurso en español, hoy. Madrid: Arco Libros.

Martín Zorraquino, M. A. y J. P. Lazaro (1999). Los marcadores del discurso. En I. Bosque y V. Demonte (dirs.), Gramática descriptiva de la lengua española (pp. 4051-4213), vol. 3. Madrid: Espasa Calpe.

Martín Zorraquino, M. A. y Montolío Durán, E. (1998). Los marcadores del discurso. Teoría y análisis. Madrid: Arco/Libros.

Montolío Durán, E. (2001). Conectores de la lengua escrita. Barcelona: Ariel.

Montolío Durán, E. (2014). Mecanismos de cohesión (II). Los conectores. En E. Montolío (dir.a), Manual de escritura académica y profesional (Vol. II) (pp. 9-92), Barcelona: Ariel.

Morales, O. y D. Cassany (2008). Interpretaciones de la atenuación en artículos de revisión de Odontología. Signos, 41(67), 299-328.

Morales, O., Cassany, D. y C. González (2008). La atenuación en artículos de odontológicos publicados en español entre 1994 y 2004: Estudio exploratorio. Revista lbérica, 14, 33-58.

Oliver del Olmo, S. (2004). Análisis contrastivo español/inglés de la atenuación retórica en el discurso médico. El artículo de investigación en el caso clínico. Tesis doctoral. Universitat Pompeu Fabra. 
Padilla García, X. A. (2008). s.v. a lo mejor En A. Briz, S. Pons y J. Portoloés (2008) Diccionario de partículas discursivas del español. En línea, http://www.dpde.es.

Parodi, G. (2008a). Géneros del discurso escrito: hacia una concepción integral desde una perspectiva sociocognitiva. En G. Parodi (coord.), Géneros académicos y géneros profesionales: accesos discursivos para saber y hacer (pp. 17-38). Chile: Ediciones Universidad de Valparaíso.

Parodi, G. (2008b). Géneros del discurso en el Corpus PUCV-2006: criterios, definiciones y ejemplos. En G. Parodi (coord.), Géneros académicos y géneros profesionales: accesos discursivos para saber y hacer (pp. 39-74). Chile: Ediciones Universidad de Valparaíso.

Parodi, G. (2008c). Géneros académicos y géneros profesionales: delimitaciones y puntos de encuentro en el corpus PUCV-2006. En Parodi, G. (coord.), Géneros académicos y géneros profesionales: accesos discursivos para saber y hacer (pp. 75-96). Chile: Ediciones Universidad de Valparaíso.

Parodi, G. (2010). Academic and Professional genre variation across four disciplines: exploring the PUCV-2006 corpus of written Spanish. Linguagem em (Dis)curso, 10, 535-567.

Parodi, G. (2015). Variation across university genres in seven disciplines: a corpus-based study on academic written Spanish. International Journal of Corpus Linguistics, 20, 469-499.

Portolés Lázaro, J. (1998). Dos pares de marcadores del discurso: en cambio y por el contrario, en cualquier caso y en todo caso. En M. A. Martín Zorraquino y E. Montolío Durán (coords.), Los marcadores del discurso. Teoría y práctica (pp. 243-264). Madrid: Arco Libros.

Portolés Lázaro, J. (2008). s.v. en cualquier caso, en todo caso . En A. Briz, S. Pons y J. Portoloés (2008) Diccionario de partículas discursivas del español. En línea, http://www.dpde.es.

Portolés Lázaro, J. (2014). Gramática, semántica y discurso en el estudio de los marcadores. En M. M. García Negroni (coord.), Marcadores del discurso: perspectivas y contrastes (pp. 202-231). Buenos Aires: Santiago Arcos.

Real Academia Española y Asociación de Academias de la Lengua Española (2009). Nueva gramática de la lengua española. Madrid: Espasa.

Regueiro, M. L. y Sáez, D. M. (2013). El español académico. Guía práctica para la elaboración de textos académicos. Madrid: Arco/Libros.

Ruiz, G. (2008). s.v. de todos modos, de todas formas, de todas maneras. En A. Briz, S. Pons y J. Portoloés (Eds.), Diccionario de partículas discursivas del español. En línea, http://www. dpde.es.

Salas Valdebenito, M. (2015). Una propuesta de taxonomía de marcadores metadiscursivos para el discurso académico-científico escrito en español. Revista Signos, 48(87), 95-120.

Santos Río, L. (2003). Diccionario de partículas. Salamanca: Luso-Española de Ediciones.

Suau Jiménez, F. (2010). La traducción especializada (en inglés y español en géneros de economía y empresa). Madrid: Arco/Libros.

Vande Koople, W. J. (1985). Some exploratory discourse on metadiscourse. College Composition and Communication, 36, 82-93.

Wiefling, F. y Montemayor-Borsinger, A. (2014). Análisis contrastivo del uso y función de marcadores discursivos en artículos de Investigación en ciencias naturales y sociales. En M. M. García Negroni (coord.), Marcadores del discurso: perspectivas y contrastes (pp. 264-276). Buenos Aires: Santiago Arcos.

Williams, J. M. (1990 [1981]). Style. Toward clarity and grace. Chicago: The University of Chicago Press. 